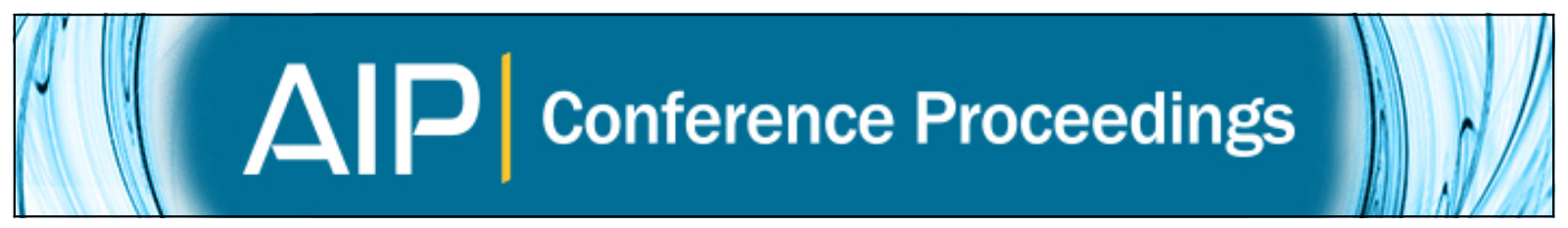

PAUT inspection of copper canister: Structural attenuation and POD formulation

A. Gianneo, M. Carboni, C. Mueller, and U. Ronneteg

Citation: AIP Conference Proceedings 1706, 200005 (2016); doi: 10.1063/1.4940649

View online: http://dx.doi.org/10.1063/1.4940649

View Table of Contents: http://scitation.aip.org/content/aip/proceeding/aipcp/1706?ver=pdfcov

Published by the AIP Publishing

Articles you may be interested in

Ultrasonic properties characterization and PAUT inspection of I-shaped CFRP component

AIP Conf. Proc. 1650, 1140 (2015); 10.1063/1.4914723

Modified formulation of a rate-dependent damage model for ductile materials

J. Appl. Phys. 107, 053513 (2010); 10.1063/1.3326939

Measurement of the hot electron attenuation length of copper

Appl. Phys. Lett. 96, 062105 (2010); 10.1063/1.3299712

Pulsed eddy current for deep metal surface cracks inspection

AIP Conf. Proc. 557, 354 (2001); 10.1063/1.1373780

Comparison between measured and predicted attenuation curves of $\mathrm{x}$-ray beams

Med. Phys. 26, 2183 (1999); 10.1118/1.598734 


\title{
PAUT Inspection of Copper Canister: Structural Attenuation and POD Formulation
}

\author{
A. Gianneo ${ }^{1, \text { a) }}$, M. Carboni ${ }^{1, b)}$, C. Mueller ${ }^{2, \text { c) }}$, and U. Ronneteg ${ }^{3, \text { d) }}$ \\ ${ }^{I}$ Department of Mechanical Engineering, Politecnico di Milano, Via La Masa 1, 20156 Milano \\ ${ }^{2} B A M$ Federal Institute for Materials Research and Testing, Berlin, Germany \\ ${ }^{3}$ SKB Swedish Nuclear Fuel and Waste Management Co., Oskarshamn, Sweden \\ a)andrea.gianneo@polimi.it \\ b)michele.carboni@polimi.it \\ c)christina.mueller@bam.de \\ d)ulf.ronneteg@skb.se
}

\begin{abstract}
For inspection of thick-walled (50mm) copper canisters for final disposal of spent nuclear fuel in Sweden, ultrasonic inspection using phased array technique (PAUT) is applied. Because thick-walled copper is not commonly used as a structural material, previous experience on Phased Array Ultrasonic Testing for this type of application is limited. The paper presents the progress in understanding the amplitudes and attenuation changes acting on the Phased Array Ultrasonic Testing inspection of copper canisters. Previous studies showed the existence of a low pass filtering effect and a heterogeneous grain size distribution along the depth, thus affecting both the detectability of defects and their "Probability of Detection" determination. Consequently, the difference between the first and second back wall echoes were not sufficient to determine the local attenuation (within the inspection range), which affects the signal response for each individual defect. Experimental evaluation of structural attenuation was carried out onto step-wedge samples cut from full-size, extruded and pierced \& drawn, copper canisters. Effective attenuation values has been implemented in numerical simulations to achieve a Multi Parameter Probability of Detection and to formulate a Model Assisted Probability of Detection through a MonteCarlo extraction model.
\end{abstract}

\section{INTRODUCTION}

According to the Swedish deposit program, the spent nuclear fuel shall be stored in cast iron inserts shielded by cylindrical copper tubes: the former gives the needed mechanical strength and the latter the corrosion resistance (Fig. 1). The estimated 100.000 years useful life, which has to be guaranteed [1], is a challenge to the integrity of the canister; hence, effort has been made, in the last years, about the quality of manufacturing process and the reliability of NDT techniques. In detail, the authors focused onto the reliability of Phased Array Ultrasonic Testing (PAUT) inspection of copper canister tubes [2] for risk assessment of nuclear waste encapsulation by means of "Probability of Detection" (POD) curves.

(a)

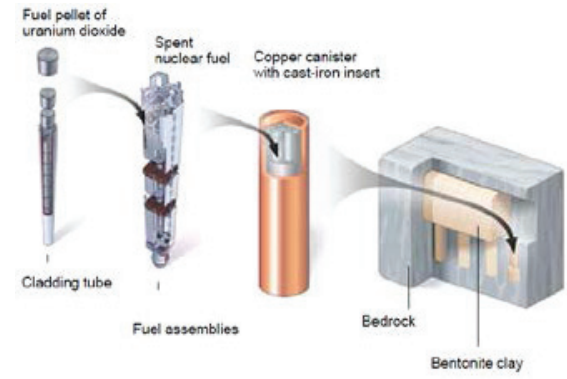

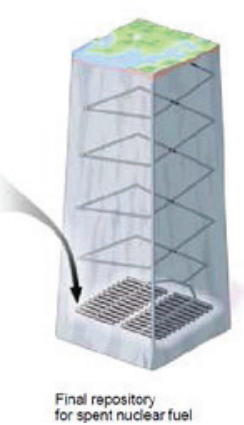

(b)

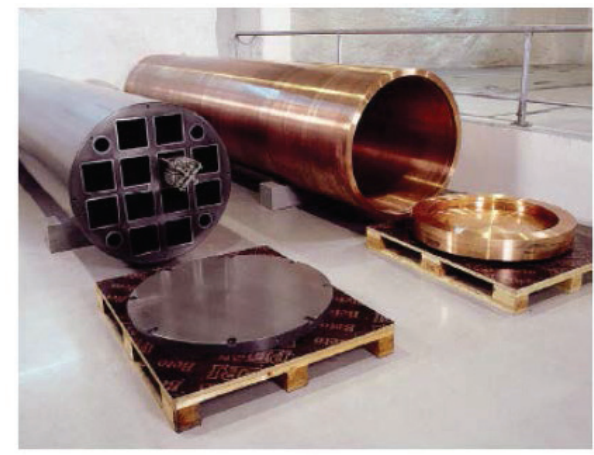

FIGURE 1. (a) Final repository structure, (b) parts representing the final disposal canister: the nodular cast iron insert (left side) and the copper tube and a copper lid (right side) [1,2]. 
Conventionally, POD curves are based onto Berens' model [3], which applies an appropriate statistical model to describe the relationship between the measured response â and the defect size a, leading to a POD curve function of one influencing parameter. Although this approach can be satisfactory in a variety of applications, for the purpose of the current NDT-reliability project, Pavlovic et al. $[4,5]$ developed a multi parameter POD approach. In this approach the depth, size, orientation of defects as well as the attenuation properties of the material were included substituting the defect size by means of numerical simulations. In Fig. 2 (a) is reported the â vs. a diagram (measured vs. modelled PAUT response) for two different regions of the T53 extruded copper tube: one with normal ultrasonic attenuation (blue curve) and one with increased attenuation (red curve). The increased attenuation can be traced back to microstructural heterogeneity. Ideally, assuming the appropriate model, the curves should follow the $45^{\circ}$ line (black one), practically, since the attenuation parameter was not yet included in the performed simulation, the data were fitted by a Least Square Estimation (LSE) [2]:

$$
\widehat{a}_{\text {measured }}=M F \cdot e^{-\left(\alpha_{0}+\Delta \alpha\right) x} \cdot a_{\text {modelled }}
$$

where $\widehat{a}_{\text {measured }}$ is the censored measured amplitude, $a_{\text {modelled }}$ is the simulated amplitude without attenuation, MF the factor to calibrate the simulated data to experiments and $\alpha$ is the actual attenuation coefficient, composed of $\alpha 0+$ $\Delta \alpha$. The former from the difference between two consecutives Back Wall Echo (BWE1 and BWE2) and the latter an extra amount from the frequency shift inside the material. The results of the LSE are shown in Fig. 2 (b), where the $\alpha$-renormalized curves follow the ideal curve; Fig. 2 (c) displays $\alpha$ values for both the low and high attenuating regions of the T53 tube.

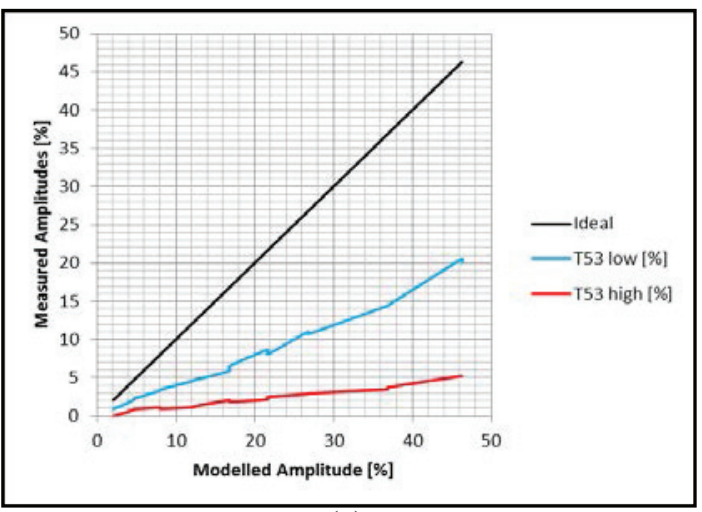

(a)

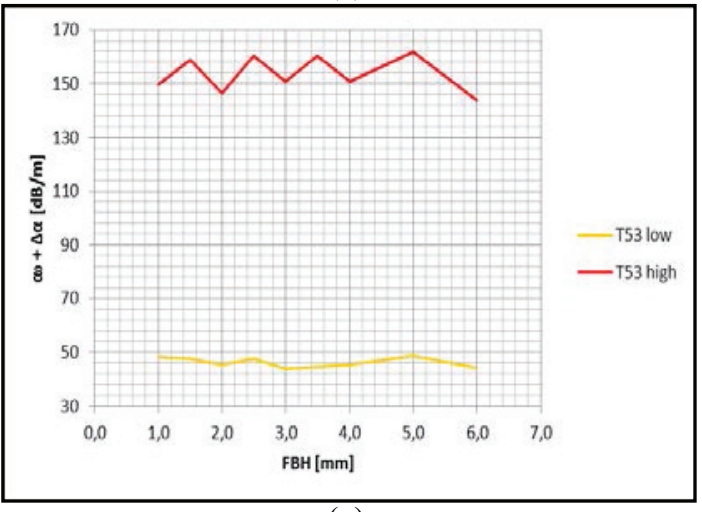

(c)

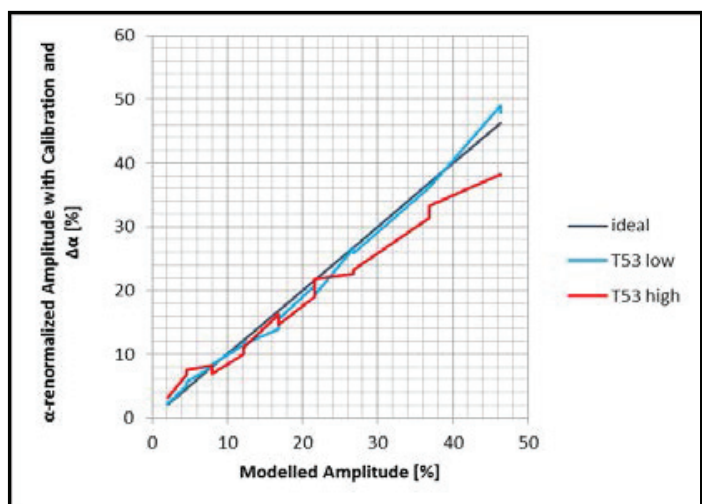

(b)

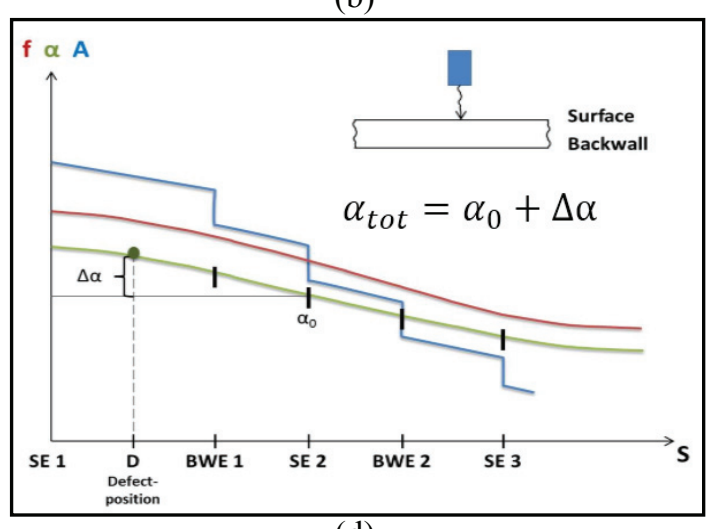

(d)

FIGURE 2. a) â vs. a diagram for T53, b) $\alpha$-renormalized measurements with model calibration and $\Delta \alpha$, c) estimated $\alpha$ values, d) qualitative behavior of attenuation coefficient, mid frequency and amplitude as a function of UT travelling path respectively described by green red and blue curve [2]. 


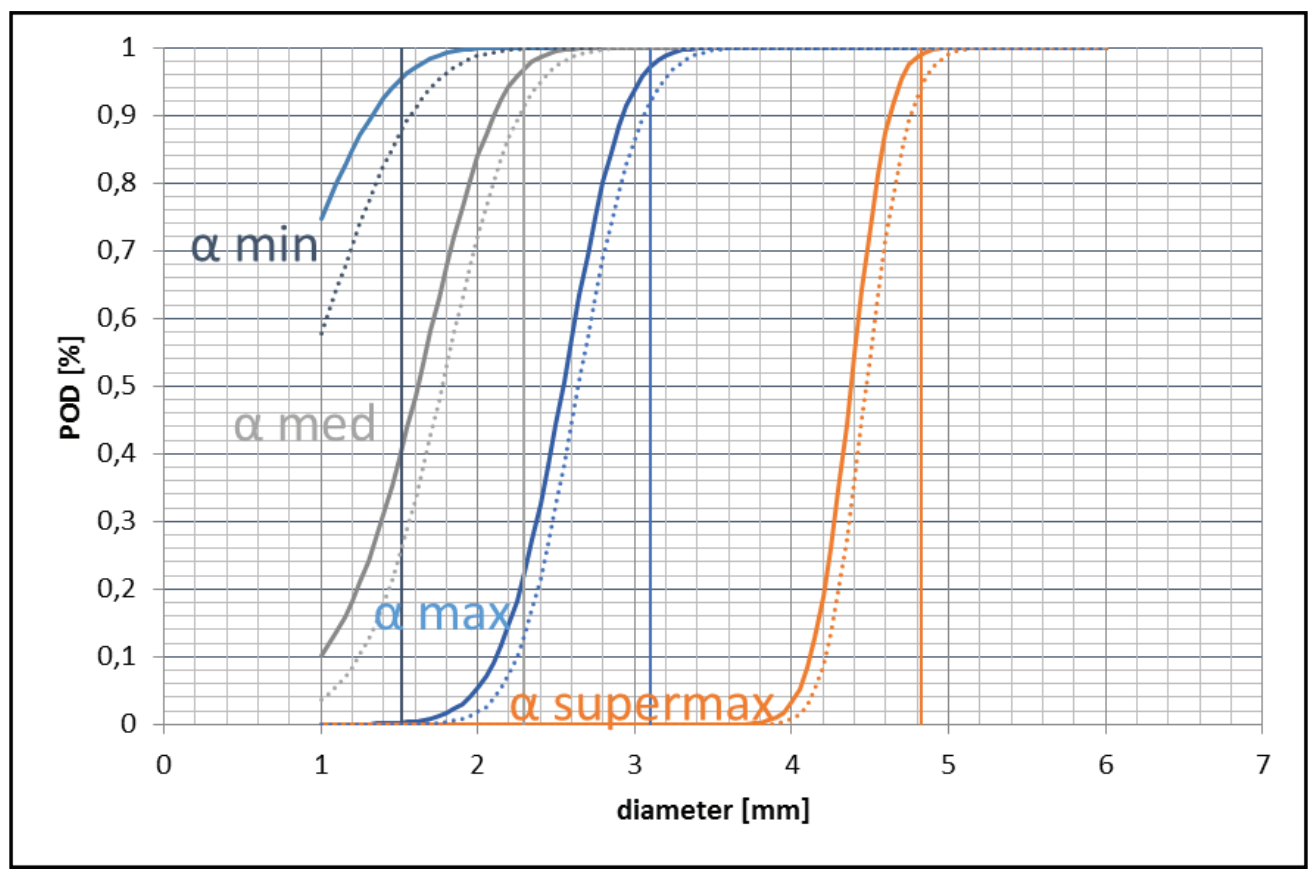

FIGURE 3. Summary of the calculated POD curve from Multi Parameter approach for higher $\alpha$ values, as a function of FBH diameter with d90/95 values [2].

After the multi-parameter POD curve is established, it can be split to the individual dependencies as depth, diameter and attenuation properties: Fig. 3 gives detectable flat bottom hole (FBH) sizes, as represented by the corresponding $d_{90 / 95}$ values, varying from less than $2 \mathrm{~mm}$ for the low attenuating material up to $4.8 \mathrm{~mm}$ for the high attenuating one.

Anyway, a deep data analysis turned out that the attenuation is not only dependent on the surface position of the copper tubes, but also on the depth position as the actual grain size distribution causes a low pass filtering of the ultrasonic signal. The frequency shift between the surface echo (SE) and BWE1 varied from $12 \%$ for the lowest attenuating material up to $34 \%$ for the highest one. Consequently, the differences between BWE2 and BWE1 were not sufficient to determine the local attenuation [2], which affects the UT-wave responsible for defect detection, as shown qualitatively in Fig. 2 (d). The remaining difference in attenuating coefficient was determined by a LSE approach assuming that the modelled amplitude and the measured amplitude should form a $45^{\circ}$ line.

\section{ULTRASONIC CHARACTERIZATION OF ATTENUATION PROPERTIES}

To confirm the approach and results developed by Müller et al. [2] and to achieve a deeper insight about the actual inhomogeneity along the depth axis, in the present paper experiments onto samples cut from an extruded full size T53 copper tube are presented.

\section{Effect of Grain Size Distribution on PAUT Inspection}

Since it's well known from the literature $[6,7]$ the influence of the frequency/wavelength, mean grain size, grain size distribution (GSD) on the UT attenuation behavior in polycrystalline materials, two step wedges for each of the two principal stress directions of the T53 extruded copper tube were machined as showed in Fig. 4, for a totality of four specimens. The nomenclature T53-XXX-YYY stands for the axial and circumferential tube position as reported in Table 1. 


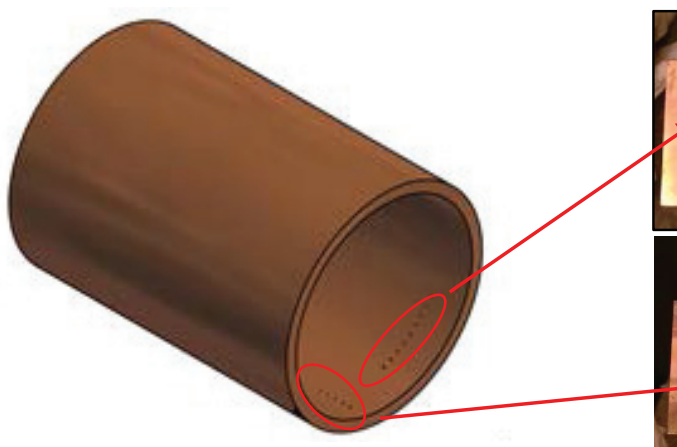

(a)

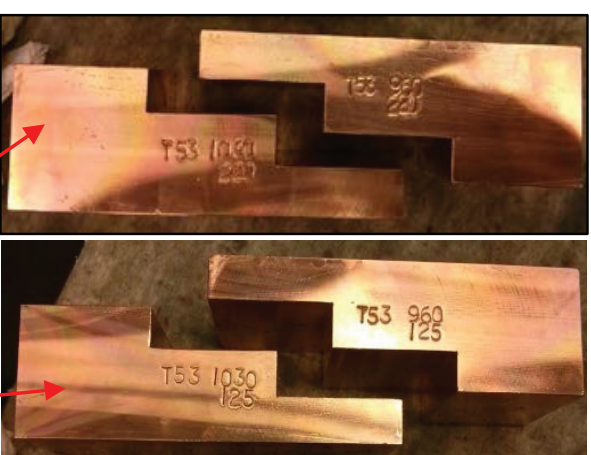

(b)

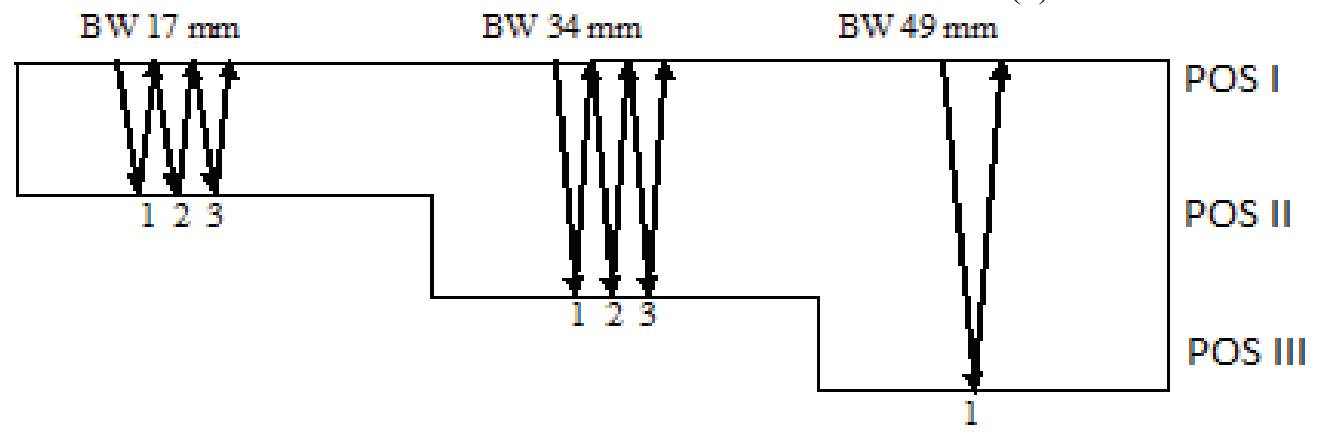

(c)

FIGURE 4. (a), (b) Machined step wedges from the extruded copper canister T53 from the low attenuating area (top) and from the high attenuating area (bottom); (c) location of measured GSD.

TABLE 1. T53 Step Wedge specimens [2]

\begin{tabular}{lccccccc}
\hline \multicolumn{1}{c}{ Identity } & $\begin{array}{c}\text { Circ. } \\
\text { Pos. }\left({ }^{\circ}\right)\end{array}$ & $\begin{array}{c}\text { Axial pos } \\
(\mathrm{mm})\end{array}$ & $\begin{array}{c}\text { Planar } \\
\text { surface }\end{array}$ & $\begin{array}{c}\text { Thickness } \\
\text { step 1 (mm) }\end{array}$ & $\begin{array}{c}\text { Thickness } \\
\text { step 2 (mm) }\end{array}$ & $\begin{array}{c}\text { Thickness } \\
\text { step 3 (mm) }\end{array}$ & Attenuation \\
\hline T53 960-125 & 125 & 960 & Outer dia. & 17 & 34 & 49 & High \\
T53 1030-125 & 125 & 1030 & Inner dia. & 17 & 34 & 49 & High \\
T53 960-220 & 220 & 960 & Outer dia. & 17 & 34 & 49 & Low \\
T53 1030-220 & 220 & 1030 & Inner dia. & 17 & 34 & 49 & Low \\
\hline
\end{tabular}

The GSD along the depth, at the fixed positions POSI, POSII and POSIII highlighted in Fig. 4 (c), was provided according to the "lineal intercept method" suggested by ASTM E112-13 [8]. Fig. 5 (a), (b) points out the most appropriate fitting of the data according to the lognormal distribution, whereas in Fig. 5 (c), (d) the standard deviation and mean grain size diameter of the respective distributions are presented.

The analysis of the aforementioned GSDs and their statistical synthesis features points out the extruded copper tube is anisotropic in the two different areas: in the T53 Low sample, the grain size distribution is almost homogeneous along the depth with a grain size diameter $D=80 \pm 70[\mathrm{um}]$. On the contrary, in the T53 High sample the GSD is showing a positive gradient along the depth regarding both $\bar{D}$ and $\sigma$. It's interesting to point out that, in POSI T53 High, the same GSD of the T53 Low is observed.

Qualitatively, the considerations previously highlighted in terms of GSD can be also evidenced through the C-scan analysis of the specimens reported in Fig. 6 (a) for step-wedges T53 Low and High, respectively. The here involved PAUT configuration, is similar to the one adopted at SKB facilities for copper tube inspection: it consists of an IMASONIC $3.5 \mathrm{MHz}, 128$ elements, Linear Phased-Array probe, with 16 elements aperture and a focal distance of $40 \mathrm{~mm}$. Coupling is guaranteed by a complete immersion tank with a $30 \mathrm{~mm}$ water column. Coherently with [2], this will be referred to as "UT11 PAUT" setup in the following. 


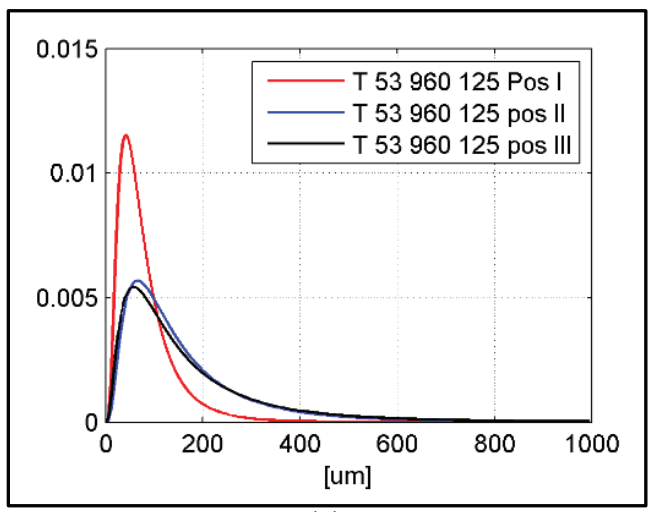

(a)

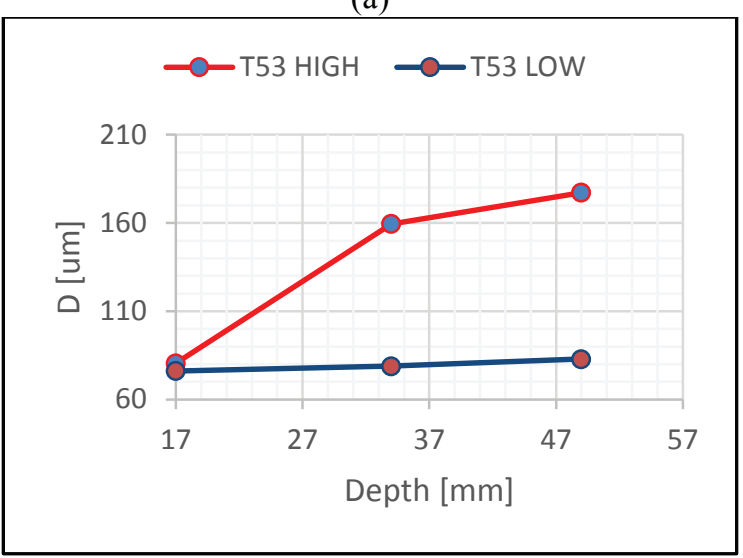

(c)

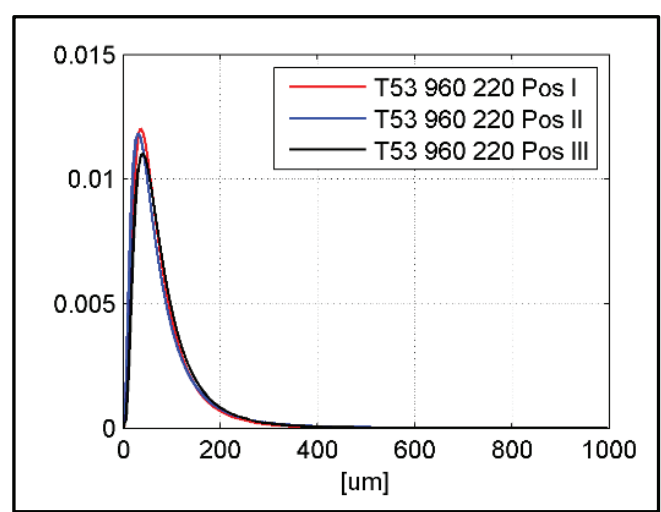

(b)

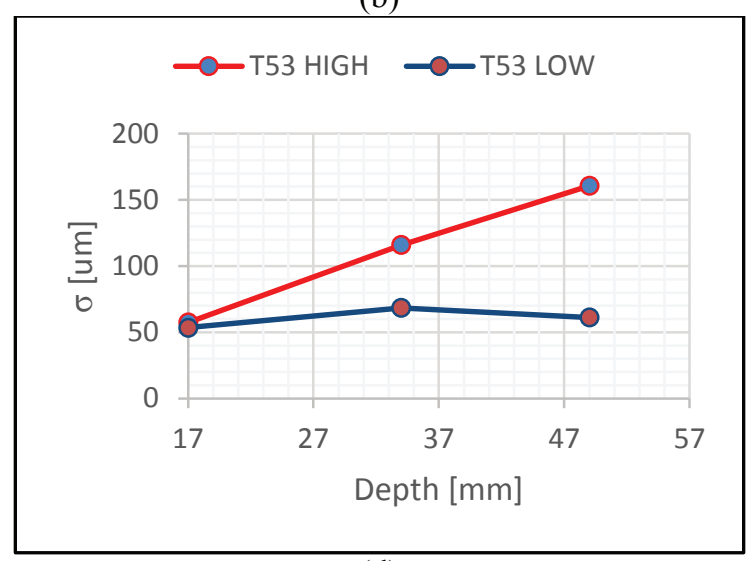

(d)

FIGURE 5. (a), (b) log-Normal grain size distribution for T53 High and T53 Low, (c), (d) mean grain size and std. deviation along the depth.

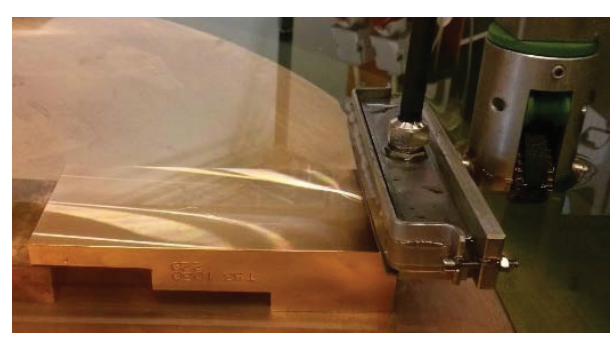

(a)

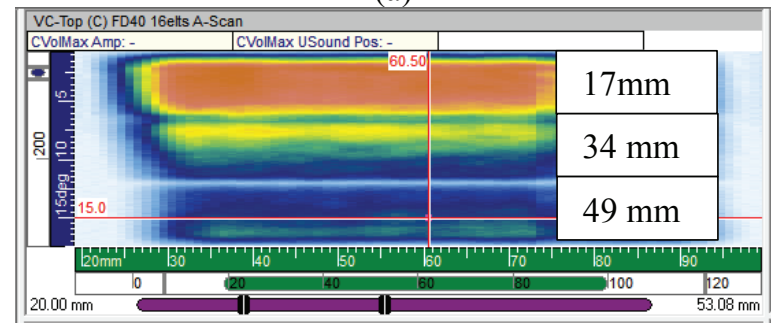

(c)

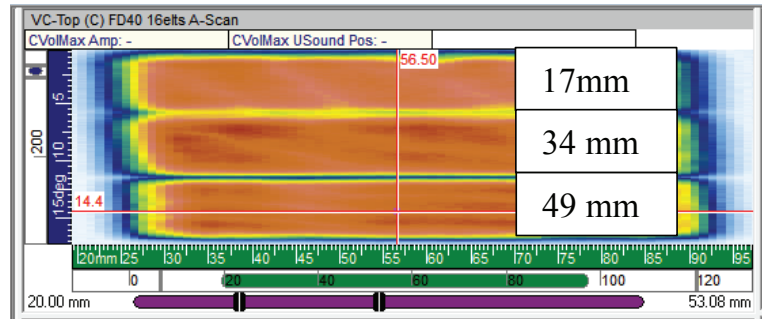

(b)

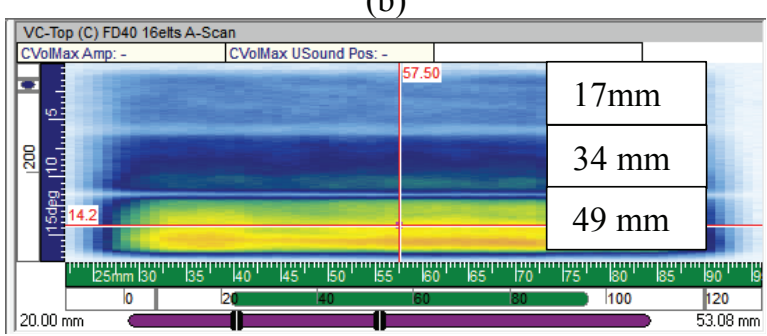

(d)

FIGURE 6. (a) PAUT inspection setup for T53 step wedges, C-Scans (b) T53 960-220, (c) T53 960-125 with the outer diameter as planar surface, (d) T53 1030-125 inner diameter as planar surface.

Moreover, the heterogeneity of GSD plays an important role in the direction of inspection: the response from BWEs, with a wall thickness of $49 \mathrm{~mm}$, will be very different from the outer or inner surface of the tube even if the GSD is the same. Comparing Fig. 6 (c) and (d), the GSD is the same, but the order of interaction is reversed. Details will be given in the following section, but, to give an idea, starting with the same SE information, if the sound beam 
firstly interacts with the finest GSD it will preserve the higher frequencies in its spectrum, leading to high attenuation values. On the contrary, interacting immediately with the coarser structure it will result in a low pass filtering of the highest frequencies and consequently lower levels of attenuation. The key point here is the information preserved in the spectrum domain.

\section{Extraction of Attenuation Values}

In completing the research, especially for heterogeneous media, a more effective method, of using the back wall echo information, is moving from the time domain to the frequency one analysing the spectrum (shape, central frequency, bandwidth ...). Thus, from the B-scans and C-scans acquired by the Ultravision 3.6 R2 Zetec Software, regarding the setup above-mentioned in Fig. 6 (a), A-scans were exported in a text file and each BWEs post processed according to the Fast Fourier Transform FFT by Matlab R2013b, as schematized in Fig. 7.

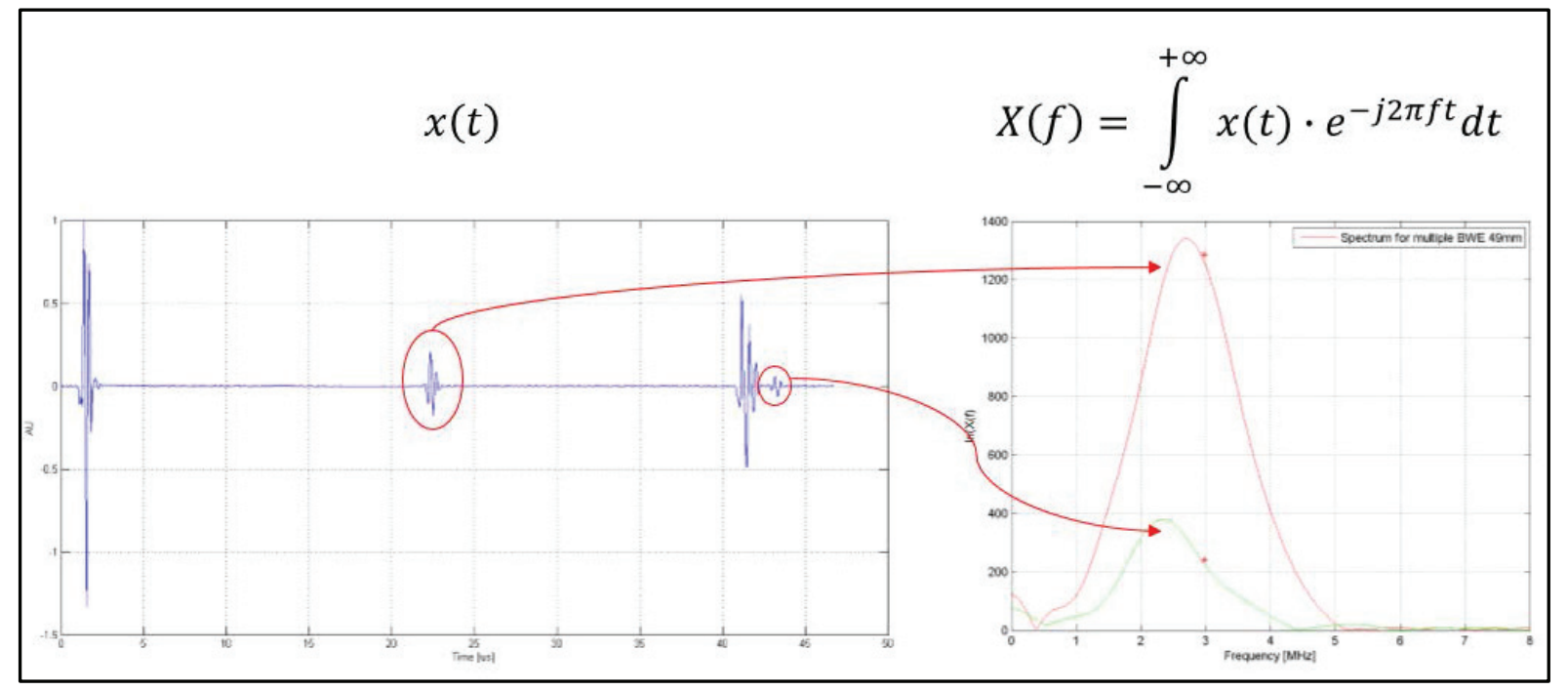

FIGURE 7. PAUT A-scan from the $49 \mathrm{~mm}$ thickness T53 Low step-wedge and estimated spectrum of the involved BWEs.

The analyzed information from the frequency domain the T53 step-wedge depth of $49 \mathrm{~mm}$ is reported in Fig. 8 and in Table 2; the real part of the spectrum and the frequency shift are illustrated, starting from the SE, for consecutive BWEs.

TABLE 2. Central frequency shift for T53 49mm step-wedge.

\begin{tabular}{cccc}
\hline & SE Frequency (MHz) & BWE1 Frequency (MHz) & BWE2 Frequency (MHz) \\
\hline T53 960-220 49mm & $3.17 \pm 0.02$ & $2.87 \pm 0.01$ & $2.58 \pm 0.06$ \\
T53 1030-220 49mm & $3.17 \pm 0.02$ & $2.94 \pm 0.01$ & $2.71 \pm 0.07$ \\
T53 $960-12549 \mathrm{~mm}$ & $3.17 \pm 0.02$ & $2.54 \pm 0.02$ & $1.88 \pm 0.21$ \\
T53 1030-125 $49 \mathrm{~mm}$ & $3.17 \pm 0.02$ & $2.78 \pm 0.01$ & \\
& & & $2.46 \pm 0.04$ \\
\hline
\end{tabular}




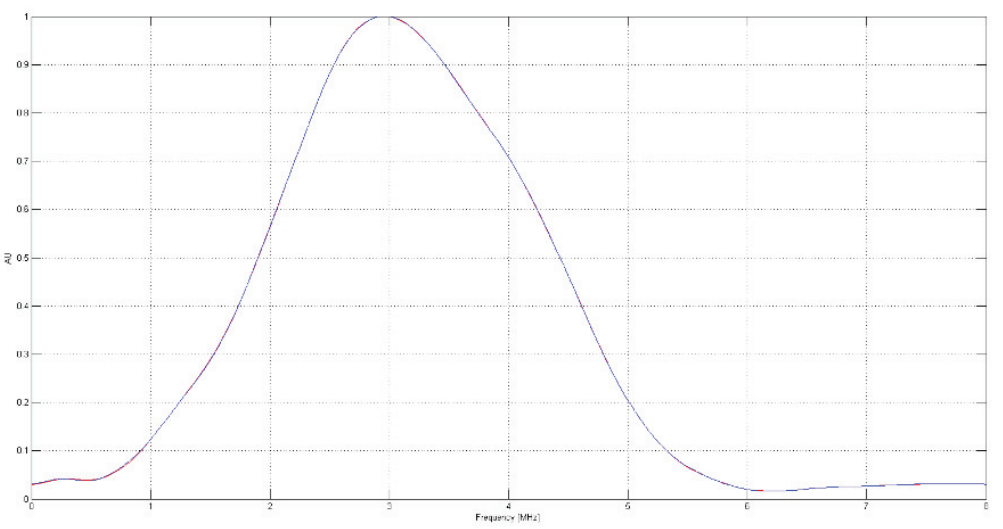

(a)

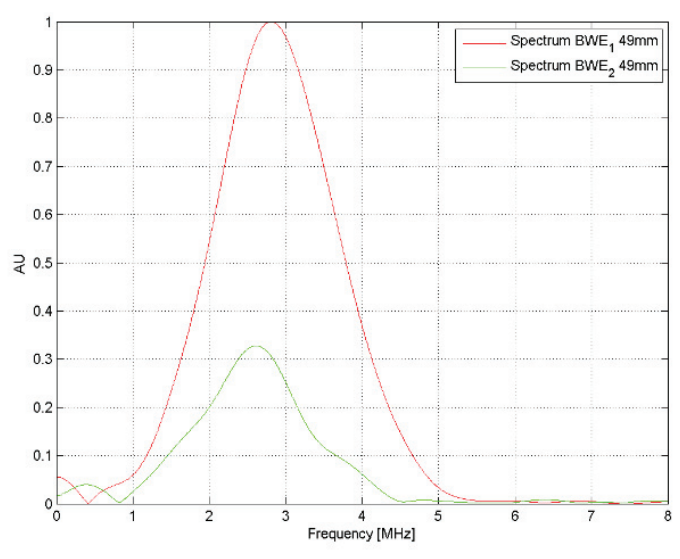

(b)

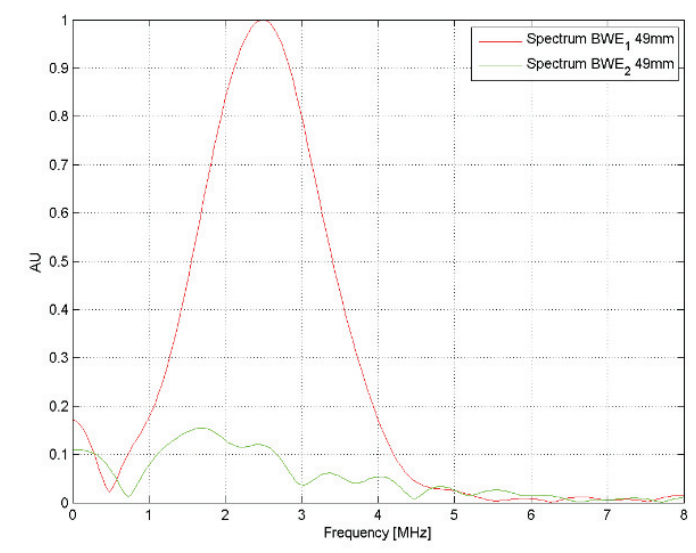

(d)

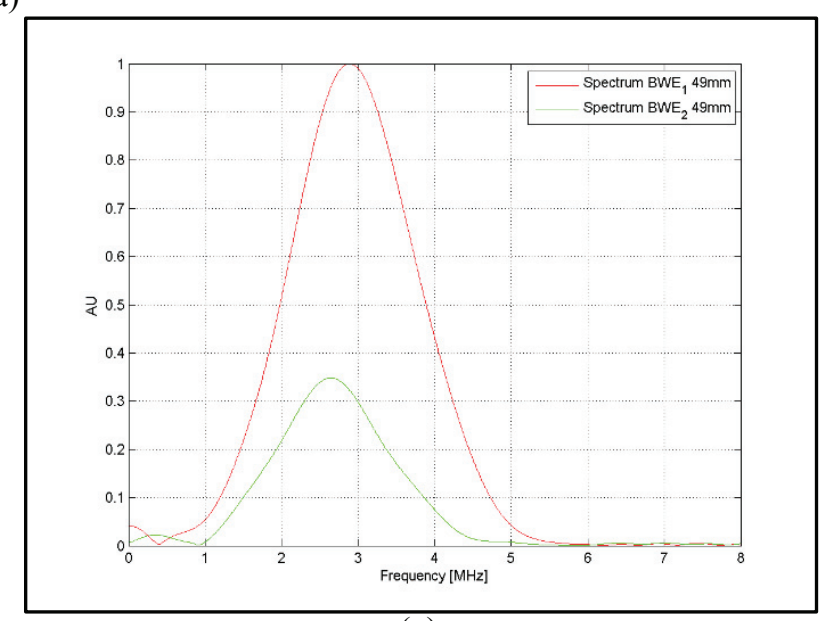

(c)

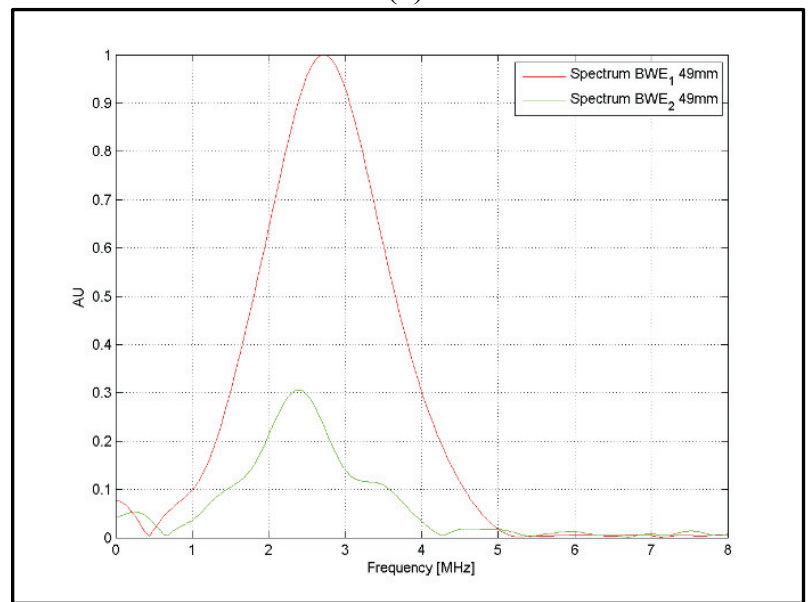

(e)

FIGURE 8. Spectrum from: (a) Surface Echo, T53 (b) 960-220 (c) 1030-220 (d) 960-125 (e) 1030-125; for (d) the SNR is about 6dB; PAUT setup UT11; red line BWE1, green one BWE2.

From the spectra reported in Fig 8, the low pass filtering effect is clearly seen: the Gaussian pulse shape is still conserved, but the higher frequencies are more attenuated than the lower ones, giving, as a result, a downward shift of the central frequency. This effect is present for both T53 Low and High, but it is much more pronounced for the latter one (Fig. 8 (d), (e)). In particular, as it could be expected, the homogeneity of T53 Low gives the same BWE 
frequency content independently from the way of inspection, whereas the direction of inspection, i.e. from the outer or inner surface, gives a different response as clearly notable comparing Fig. 8 (d) and (e). That implies different interaction and scattering responses from possible defects/discontinuities, since the intrinsic characteristic of the interacting wave in terms of central frequency and bandwidth information is different.

Clearly, a frequency shift of $14 \%$ from the nominal $3.5 \mathrm{MHz}$ gives negligible difference in the scattered amplitude, on the contrary, high-attenuated material, as copper tubes from pierce and draw manufacturing process [2], can exhibit a shift up to $40 \%$ and hence significantly influenced scattered amplitudes.

In addition, the broadband pulse (the frequency content of SE at $-6 \mathrm{~dB}$ belongs to [1.9; 4.4] MHz), allowed defining a monochromatic apparent attenuation $\hat{\alpha}(f)$ as:

$$
\alpha(f)=\frac{d B\left(\frac{X(f)_{B W E(i)}}{X(f)_{B W E(i+1)}}\right)}{2 L}
$$

where $X(f)_{B W E(i)}$ is the amplitude at the fixed frequency $\boldsymbol{f}$ of the i-th BWE, and $2 \mathrm{~L}$ is the propagation distance (twice the thickness). Once corrected by transition losses and beam spreading, the results can be used to build up a discrete attenuation curve describing the trend of attenuation against the frequency for each GSD, or, fixed a frequency, to describe the GSD evolution along the depth, as illustrated for the more interesting T53 High Step-Wedges.

Fig. 9 (a), (b) point out, regarding the inspection both from the outer and inner surfaces, a stable attenuation level, within $50 \mathrm{~dB} / \mathrm{m}$, whereas a growing one, illustrated in Fig. 9 (c) from $50 \mathrm{~dB} / \mathrm{m}$ up to $250 \mathrm{~dB} / \mathrm{m}$ in agreement with the GSD gradient of Fig. 5 (a). On the contrary, inspecting from the inner surface, Fig. 9 (d), the highest $\alpha$ values are achievable within the first skin millimeters (coarser GSD), and the lowest one regarding the overall thickness of 49 $\mathrm{mm}$. The latter is the superposition of all GSD along the depth. This trend is exactly opposite of the former and has to be attributed to exactly flipped GSD order as showed. Anyway, it is interesting to highlight that the same inspected thickness, i.e. $49 \mathrm{~mm}$, gives a higher level of attenuation or a lower one depending on the order of interaction with GSD, in this case $250 \mathrm{~dB} / \mathrm{m}$ against $80 \mathrm{~dB} / \mathrm{m}$, although the number of interactions within the depth is the same.
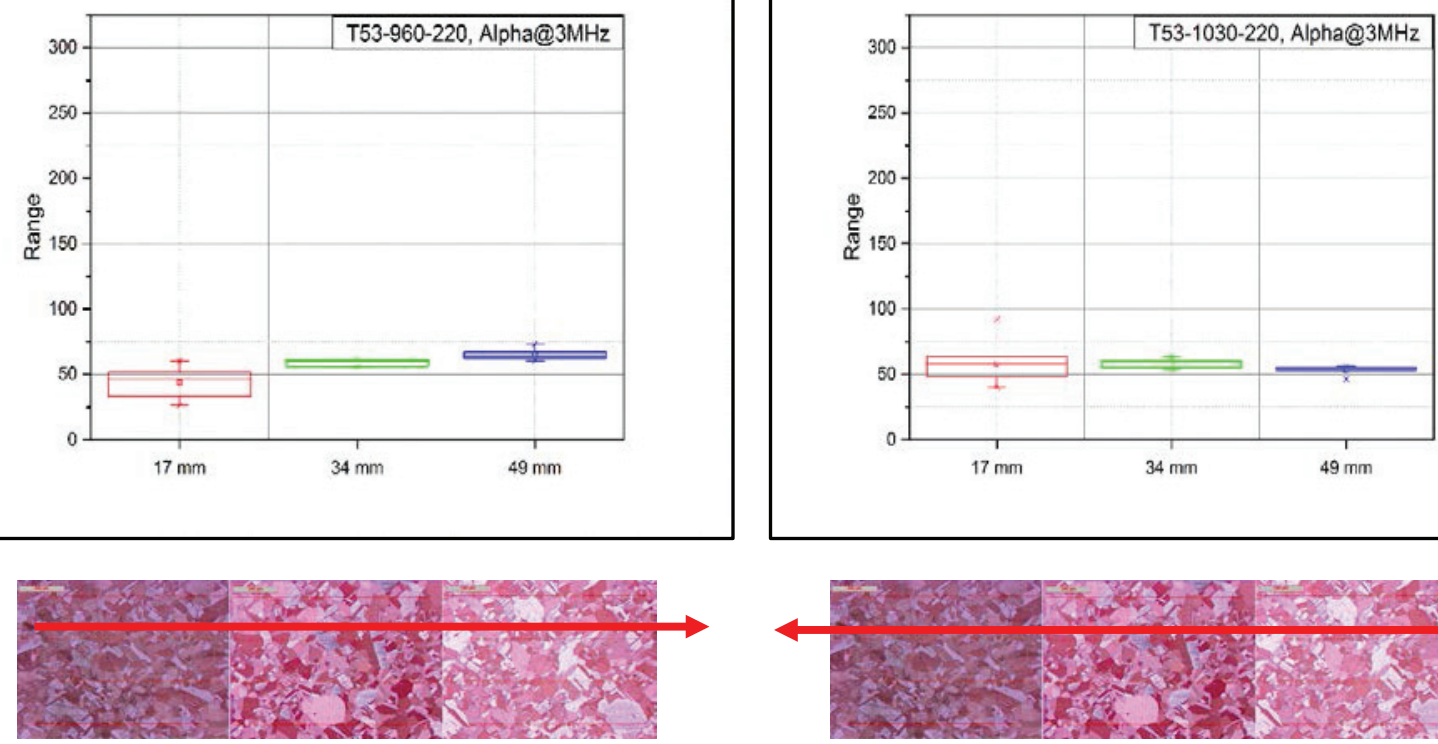

(a)

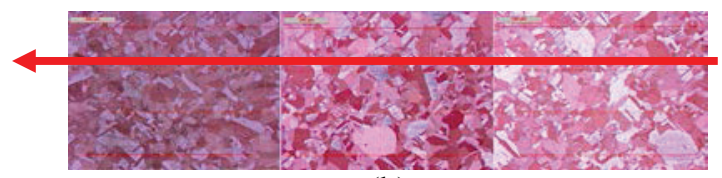

(b) 

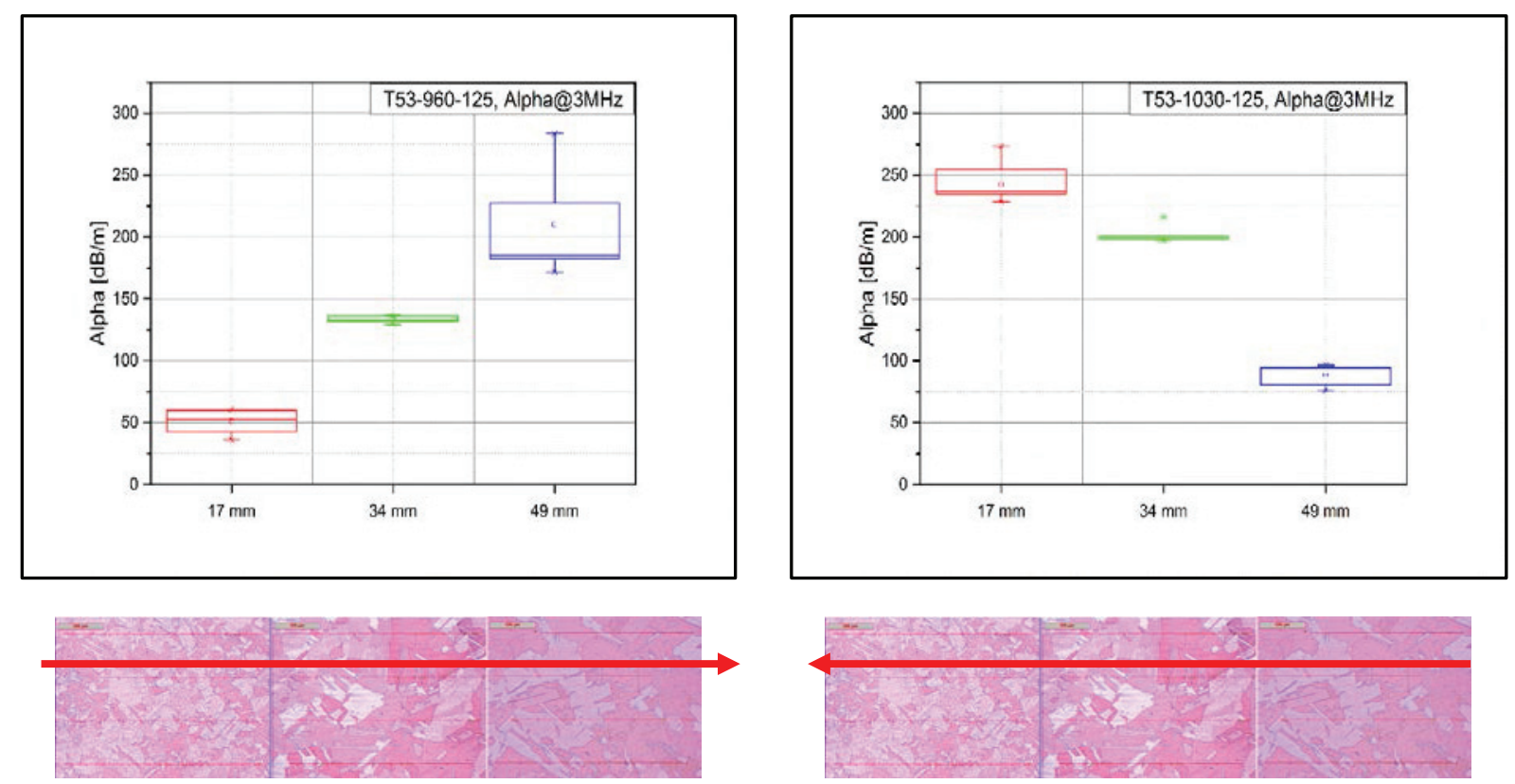

(c)

(d)

FIGURE 9. Attenuation $\alpha(\mathrm{f})$ as a function of the depth for T53 copper tube: (a) T53 960-125, (b) T53 1030-125; frequency fixed at $3 \mathrm{MHz}$.

\section{PAUT INSPECTION CAPABILITY}

The capability of the developed phased array ultrasonic technique has been investigated on copper tube material with two different levels of ultrasonic attenuation along the hoop and axial directions.

The investigation has been mainly performed by the use of the multi-parameter POD methodology [2, 4, 5], which allows several influencing factors to be included. In this work, the main parameter were the varying attenuation in the copper tubes and the main challenge was to incorporate the physics of the ultrasonic wave in the copper tube material with various and uniform ultrasonic attenuations.

Within this framework, a multi-parameter approach is to be preferred for the analysis of phased array ultrasonic focused inspection technique for complex parts, because the POD is a forecast or interpolation means, on the basis of the underlying physical relations, and is most correctly included, when the modelled amplitude is applied for this exact application case. This approach, furthermore, provides the advantage to include further parameters, such as the depth position or the angle of the defect, in the existing model with much lower experimental effort than producing an empirical POD0 for each parameter setting and varying defect size separately. Additionally, with the aim of maximizing the efficiency of POD determination, a Full-MAPOD approach is investigated and its results compared with those from classical one parameter POD (POD-0).

\section{POD Formulation in a Multi-Parameter Perspective}

The previously described monochromatic $\alpha(f)$ values were taken as input for the Multi-Parameter POD calculations according to the procedure developed by Pavlovic $[4,5]$ : thus the a parameter, usually defined as the flaw size, is replaced by numerical UT simulation responses by means of CIVA $^{\text {nde }} 11.1 \mathrm{a}[10]$, this time directly including the attenuation behavior and the frequency shift. Numerical results were calibrated according to the first BWE of T53Low, hence without manual adaption of data and LSE. As clear from Fig. 10 (b), measured amplitudes and simulated ones are in good agreement following an ideal $45^{\circ}$ line, while the previous approach for high-attenuated material (Fig. 11 (a)), as T53 High, shows a deviation. 


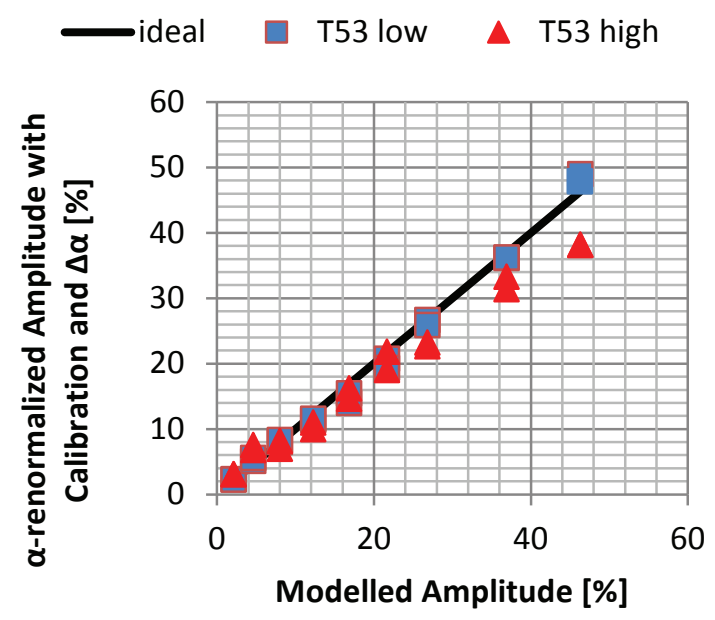

(a)

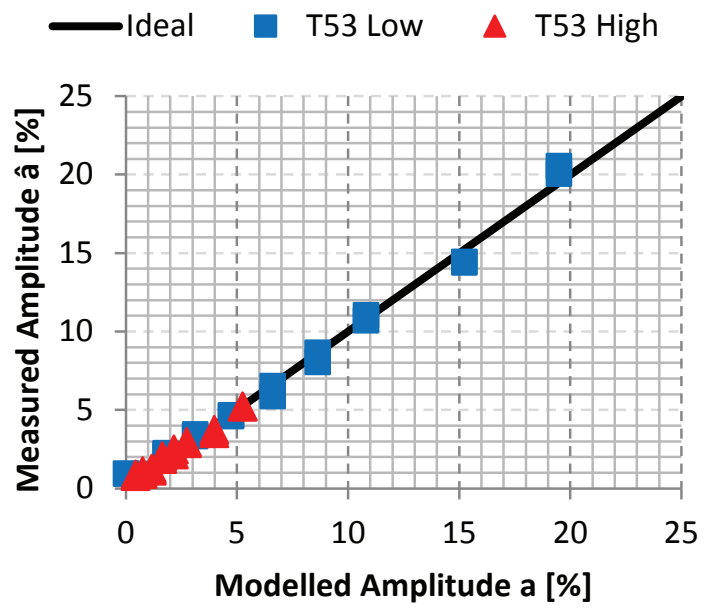

(b)

FIGURE 10. (a) â vs. $a$ diagram using the $\alpha+\Delta \alpha$ approach, (b) directly including $\alpha(f)$ values.

Additionally, our results for the MP-POD $\alpha+\Delta \alpha$ approach and full $\alpha(f)$ with the empirical one-parameter POD results were compared. The application of the one-parameter POD is possible, when the POD is considered as a function of the square of the diameter (far field behind a FBH) for each tube segment separately; a decision threshold of 3 times the mean noise level was chosen. The results are shown in Tab. 3: the d90/95 for T53 low is comparable nevertheless the procedure used for POD formulation, but for higher attenuated material the values differ more for $\alpha$ $+\Delta \alpha$, even though they are in the same order of magnitude. The fact that the sound field with its focal law and frequency shift behaviour are taken into account only in the multi-parameter approach with full $\alpha(f)$ correctly, explains why the calculation yields better results for it. This advantage will be of special interest when the defect depth is varied. In principle, this POD0 calculation supports the multi-parameter approach in both approximations. But, the advantage is seen in the higher statistical basis and the correct consideration of the PAUT focus law including frequency dependent attenuation in case of the multi-parameter data set using $\alpha(f)$. Nevertheless, the $\alpha+\Delta \alpha$ approach is an helpful mean in case a full $\alpha(\mathrm{f})$ investigation is not affordable.

TABLE 3. Results from one-dimensional POD, MP-POD $\alpha+\Delta \alpha$ and $\alpha(\mathrm{f})$

\begin{tabular}{lccc}
\hline Detectable diameter d90/95 $[\mathrm{mm}]$ & POD-One Parameter & MP-POD & MP-POD \\
& & $\alpha+\Delta \alpha$ & $\alpha(\mathrm{f})$ \\
T53 Low & 1.6 & 1.7 & 1.7 \\
T58 High & 2.7 & 2.5 & 2.8 \\
\hline
\end{tabular}

\section{Model Assisted POD}

There is effectively a possibility to diminish the experimental and cost effort maintaining the same reliability of the inspection? POD curves are based on the statistical distribution of UT responses, which, on the other hand, are controlled by numerous factors related to the adopted NDT procedure.

Today, many of such factors $[10,11]$ can be modelled and simulated by suitable physical and numerical models and MAPOD uses this possibility at its best; question still arises from the implementation of human factors [11]. Here a Full-MAPOD complete approach is established by means of numerical simulations performed by means of CIVA ${ }^{\text {nde }}$ 11.1a, where the variability source propagation, user defined by a suitable statistical distribution, follows Monte Carlo extraction [10].

Due to the highly mechanized PAUT inspection system, the MAPOD parameter here established as the main variability source is recognized as structural attenuation, following a Gaussian distribution, whose scale and location parameters come from an exponential nonlinear regression from Step-Wedges measurements. There are many other 
causes for scatter like electronic noise, coupling variations, device calibration variations, here not yet included; as a consequence the MAPOD can be seen as means to look for the trend not for the complete forecast of POD.

The effect of GSD along the depth is also included, modelling the tube as a multilayered material on the base of the experimental evidence from Step-Wedges. Additionally, the "Low-Frequency" content of PAUT probe allows for neglecting water path attenuation and the numerical model is calibrated on the base of a big reflector as the BWE1 of T53 Low Copper Canister Tube. Ideally, if the model is well established, experimental data, exactly two for each FBH diameter, must be included within numerical uncertainty: this trend is showed in Fig. 11, where, in a double logarithmic scale, the maximum amplitude UT response from experimental data is compared with the numerical one from 5 extractions for each FBH size. Having a close look, it can be highlighted that for the T53 Low the experimental points lie within the numerical scattering and the can be recognized. Whereas for the high-attenuated structure, i.e. T53 High, a close agreement is found in the FBH flaw size interval $(2.5 ; 6) \mathrm{mm}$ and a flattening behavior in the experimental response is highlighted for small FBHs. In particular, one has to expect the linear trend slope is kept constant to a power of 2 , because, from a physical point of view, the response has to increase as the square of the diameter, as pointed out for the T53 Low and T53 High simulated data. Instead, T53 High experimental data slope is rotated clockwise, underlining a lower power. This can be explained by the fact that UT response from very small FBHs diameter is within the environmental $-8 \mathrm{~dB}$ noise, superposition of electronic and material ones, thus of difficult resolution for the UT equipment.

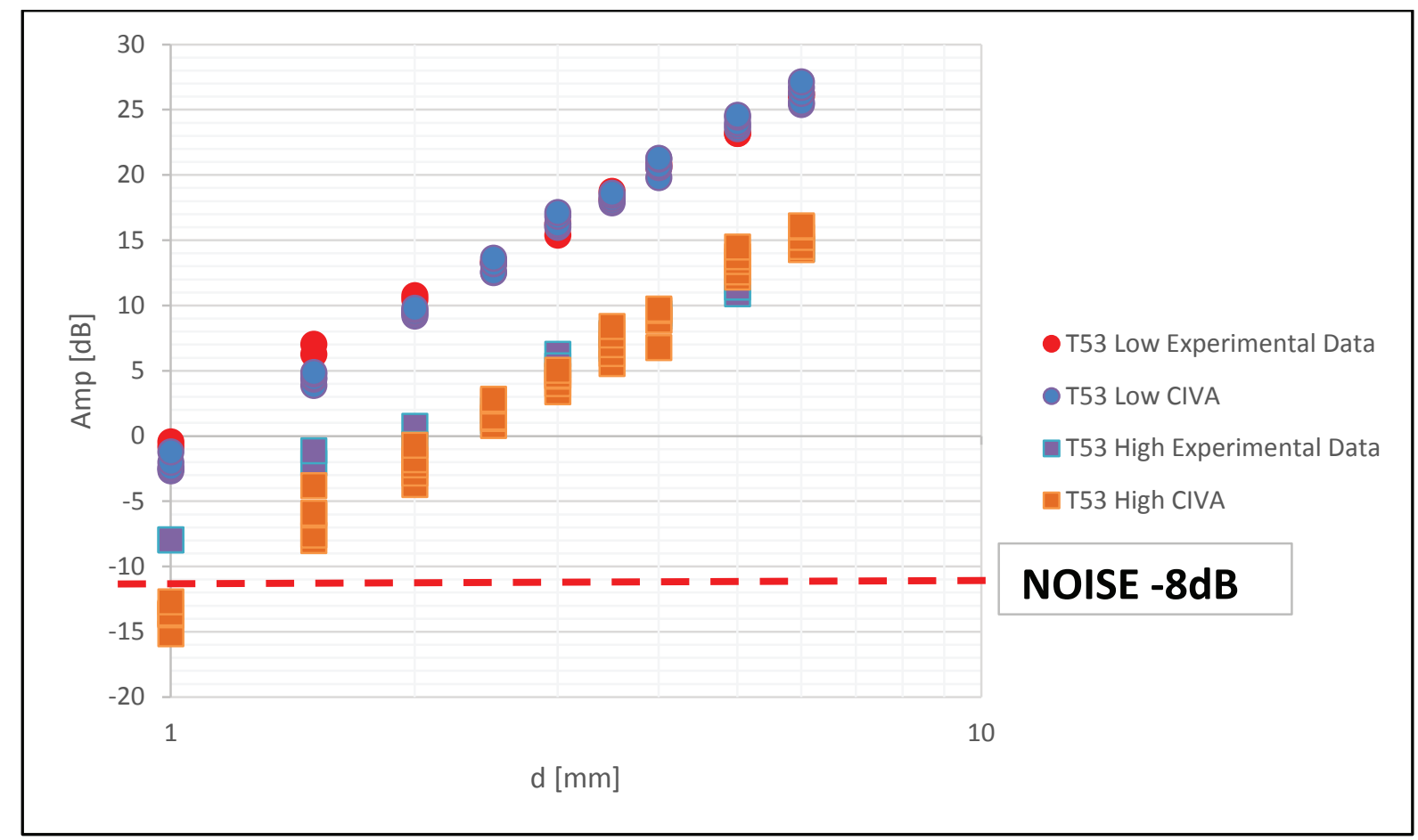

FIGURE 11. Comparison between experimental FBH response and simulated one on the base of 40 extractions.

The effect is clear also in the subsequently calculated POD curves of Fig. 12: for the T53 Low, a close agreement can be found regarding the shape, slope, confidence interval and the $\mathrm{d}_{90 / 95}$ parameter, using two Monte Carlo extractions for each FBH diameter, thus allowing the same sample size. On the contrary, although an identical d90/95 parameter and the intersection between confidence intervals, the effect of additional variability factors previously highlighted and intrinsically conditioned by the electronic equipment, and that cannot be included yet in a MAPOD simulation, lead to a shifted and counter clockwise-rotated simulated POD. 


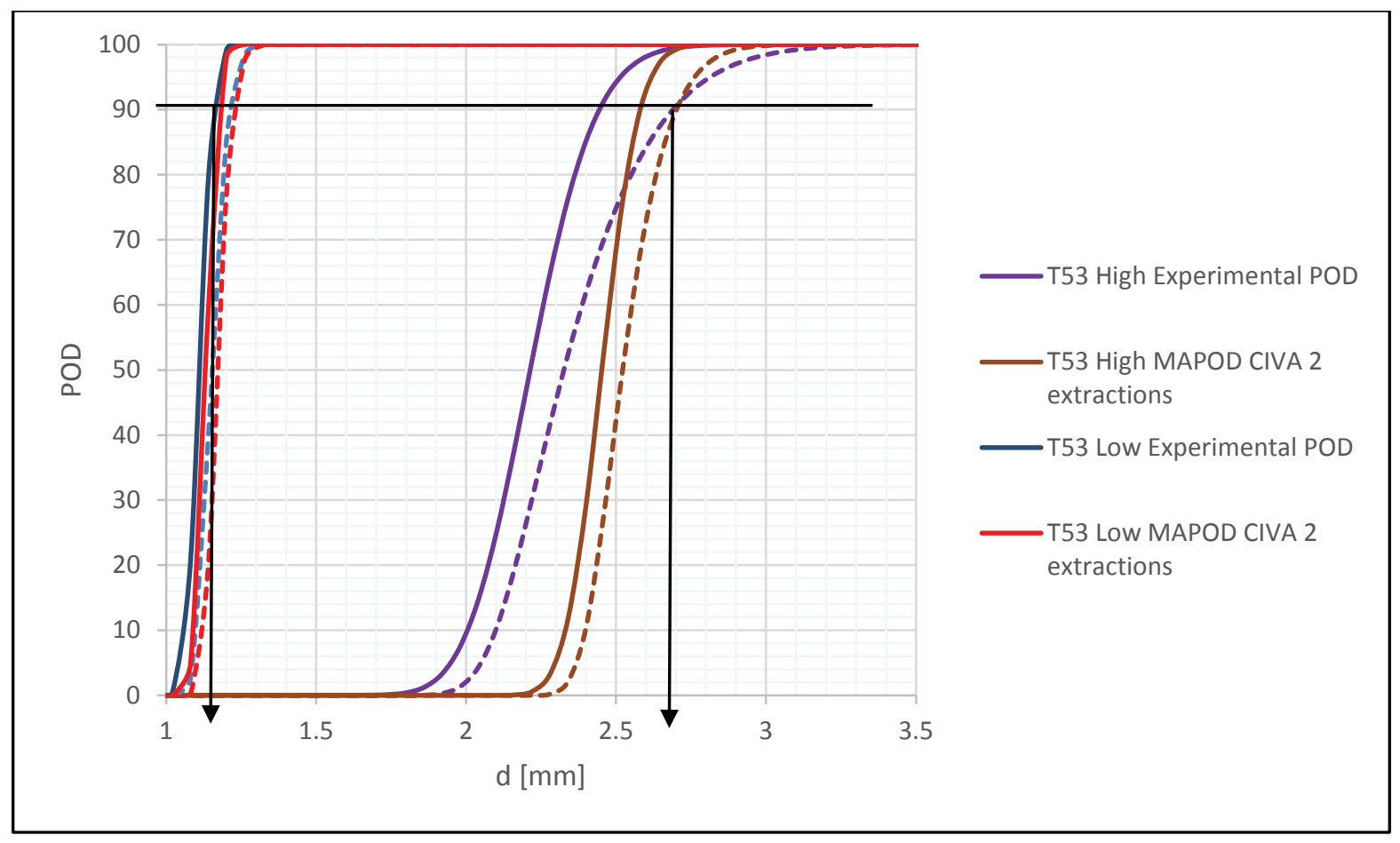

FIGURE 12. POD curves from experimental data and from a Full-MAPOD approach; only two extractions were considered.

\section{CONCLUDING REMARKS}

Within the POD multi-parameter framework, the PAUT attenuation dependent detectability of copper canister has been investigated concerning an extruded anisotropic and heterogeneous copper tube material, characterized by two principal directions with various levels of ultrasonic attenuation and a Grain Size Distribution gradient along the depth. The former results showed that the attenuation and the low-pass filtering effect of UT required a manual adaption of the data to take into account frequency shift effects.

Here instead, through a detailed analysis in the frequency domain, a deeper insight into the effect of GSD against the attenuation and its influence about the UT spectrum evolution along the depth was achieved. Moreover, different inspection behaviors regarding the direction of inspection for polycrystalline materials exhibiting a GSD gradient along the depth were discussed. Currently, a database of discrete attenuation curves could be built, according to the monochromatic procedure here presented, for different materials, and consequently dissimilar GSD along the depth, in order to directly include, through numerical simulation, the attenuation effects on the UT spectrum content. The numerical results could then be used to improve the PAUT inspection procedure and to elaborate new POD curves, according to the multi-parameter procedure, without the need for manual correction of the data.

Moreover, the authors investigated the feasibility of a Full Model-Assisted Probability of Detection (MAPOD) methodology: in some cases there is effectively a possibility to diminish the experimental effort maintaining the same reliability of the inspection, i.e. T53 Low, whereas "extreme" $\alpha$ values (T53 High) lead to uncertainty factors that cannot be included in simulations.

\section{REFERENCES}

1. J. Pitkänen, Posiva Oy, Inspection if Disposal Canisters Components, POSIVA 2012-35 Report, (December 2013).

2. C. Müller, T. Heckel, P. Brömel, M. Pavlovic, U. Ronneteg, 'Attenuation dependent detectability at ultrasonic inspection of copper', SKB, 2014.

3. A. P. Berens, NDE Reliability Data Analysis - Metals Handbook Volume 17, 9th Edition: Non-destructive Evaluation and Quality Control, ASM International, OH, (1989). 
4. M. Pavlovic, K. Takahashi, U. Ronneteg, J. Pitkänen, C. Müller, Multi-Parameter Influence on the Response of the Flaw to the Phased Array Ultrasonic NDT System. The Volume POD. In Proceedings of the 4th EuropeanAmerican Workshop on Reliability of NDE, Berlin, (June 24-26, 2009).

5. M. Pavlovic, K. Takahashi, C. Müller, Probability of Detection as a Function of Multiple Influencing Parameters", InsightVol 54 No. 11, pp. 606-611, November 2012.

6. R. L. Smith, The effect of grain size distribution on the frequency dependence of the ultrasonic attenuation in polycrystalline materials, Ultrasonics, Volume 20, Issue 5, pp. 211-214, September 1982.

7. E. P. Papadakis, Revised Grain-Scattering Formulas and Tables, Journal of the Acoustical Society of America, 37, pp. 703-710, November 1965.

8. ASTM E112-13, Standard Test Methods for Determining Average Grain Size.

9. MIL-HDBK-1823A, Non Destructive Evaluation System Reliability Assessment, (April 2009).

10. CIVA 11.1a User's Manual, CEA, (2015).

11. R. M. Meyer, S. L. Crawford, J. P. Lareau, M. T. Anderson, Review of Literature for Model Assisted Probability of Detection; Technical Report (PNNL \#23714) for the United States Department of Energy, (2014). 